\title{
Editorial
}

\section{Transformation in nursing education in India: Current issues}

\author{
Rakesh Sharma $^{1}$, Shweta Garadi ${ }^{1 *}$, Prasuna Jelly ${ }^{1}$, Ruchika Rani ${ }^{1}$ \\ ${ }^{1}$ College of Nursing, All India Institute of Medical Sciences, Rishikesh, Uttarakhand, India
}

\section{A R T I C L E I N F O}

Article history:

Received 05-10-2021

Accepted 11-10-2021

Available online 14-10-2021

Keywords:

International Council of Nurses (ICN)

\begin{abstract}
A B S T R A C T
This paper is aimed to understand the current issues in nursing education in India. Nursing education has gained great demand around the globe in the current scenario to call forth efficient nurse leaders and nurse executives. Nursing knowledge gained by a student is equally proportionate to competencies they develop in the future. Advancing skills, adapting to the changed curricula, implementing competitive teachinglearning methods in all nursing programs are the need of the hour now.
\end{abstract}

This is an Open Access (OA) journal, and articles are distributed under the terms of the Creative Commons Attribution-NonCommercial-ShareAlike 4.0 License, which allows others to remix, tweak, and build upon the work non-commercially, as long as appropriate credit is given and the new creations are licensed under the identical terms.

For reprints contact: reprint@ipinnovative.com

\section{Introduction}

International Council of Nurses (ICN), in its statement on nursing education, nursing practice and nursing service, and the social and economic welfare of nurses, believes that a sound national system of nursing education is essential for quality nursing care. ${ }^{1}$ Nursing education is looking forward to build not just intellectuals but also well-qualified professionals to address the collective needs of transforming society, focusing on best of the best quality care based on scientific evidence. ${ }^{2}$ Evidence-based education modules in the curriculum that aims to improve the critical thinking and decision-making skills of nurses can help in fulfilling this need. $^{3}$

In this context, strategies in nursing education have made an incredible shift from idealism to pragmatism, including students to inquire and search for the best evidence rather than following the traditional learn and grow the concept. ${ }^{4}$ Accessibility of quality education to students further can be provided by maintaining a balanced exposure of theory and clinical areas. For this, we need proficient nurses' workforce who can accept the shift happening in educational policy

\footnotetext{
* Corresponding author.

E-mail address: shwetagaradi12@gmail.com (S. Garadi).
}

and exhibit interest to learn and discover new technological advances.

It is imperative to look into the challenges existing and arising in nursing education. The ICN Nursing Education Network addresses worldwide nursing educational issues. The preparation of a diverse 21 st-century nursing workforce is a critical priority to cope with issues including international migration, a global healthcare and the worldwide nursing shortage. ${ }^{5}$ The authors of this paper would ideally suggest there are numerous ways to be adapted to overcome the issues in nursing education not only just by integration of theory into clinical practice but also through evidence-based practice, excellent and innovative teaching-learning strategies along with appropriate training and enhancement of teachers and nursing educators on learning skills and updates.

\section{Current Issues}

\subsection{Alignment of nursing practice with theory}

Though the nursing theory is in congruence with the practice, the integration of both should occur at a rapid pace, and educators should be able to adapt to this rapidity 
to set out the best outcomes from the learners as well. Still, inadequacies are shown up in this specific area as a considerable challenge. Report of Institute of medicine in 2011 goals to double the number of nurses with a doctorate program by $2020 .{ }^{6}$ The report also calls for higher levels of nursing education and preparation and is pushing for enhanced access to innovative programs. Choosing the right student for the nursing profession ensures greater job compatibility and thus improves further performance and outcomes in the future. ${ }^{7}$

To address this challenge, the clinical environment should be facilitated with safety causing to manage well emotional reactions of nursing students and increasing their self-confidence would be of greater help. ${ }^{8}$ Continuing mentoring regardless of a student's need for an emotional reaction and peer learning would even play better roles to create a supportive environment. Many more skills labs should be opened in the nursing institutes to facilitate a safe environment to practice on mannequins to finer clinical skills. Padhila et al. stated from their randomized control trial that nursing students had shown better knowledge retention, clinical reasoning and satisfaction with the learning experience. ${ }^{9}$

\subsection{Abrupt change in curriculum}

Landmark change happened in modifying into the competency-based curriculum with new elements added in it. Time for self-directed learning is considered an environment that has to be provided for the same. Implementation of Cumulative Grade Point Average (CGPA) for marking academic excellence and semester system has gained much attention of professional workforce. $^{10}$ Such paradigm shifts need to prepare and train well teachers for better implementation of the curriculum in place. ${ }^{11}$

The grading system was invented long ago by William Farish in 1972 at Cambridge University of England. ${ }^{11}$ It has taken several decades to be in the limelight and finally adopted by the nursing profession now. The sudden acceptance of this change may raise many concerns such as confusion, lack of preparedness, lack of readiness for innovative methods and lack of proper resources and facilitators.

\subsection{Technological advancements and digital health}

Technology plays a significant role in our daily professional life, including education, patient care, research and administrative levels. In nursing education, skill-based techniques using the operation of simulation pads and mannequins need expertise to handle them carefully; in research, updated databases and search strategy and even online submission of research papers and deliberate use of statistical packages is an essential skill to be developed by a nurse researcher. Using smart boards in classrooms with multifaceted benefits still needs repeated demo sessions to those who handle it. ${ }^{12-14}$

Pandemic has made everything digital, including health care by telemedicine and telenursing services focusing on competencies in nursing informatics. Informatics needs though highly efficient trainers to train end-users, it makes healthcare democratized and easily accessible in the ecosystem. Cutting-edge medical technology is invading space in the current scenario by creating smart cloud-based predictive health care applications. ${ }^{15}$

\subsection{Research with a broader scope}

Nursing research decades ago was mainly concentrating on assessing the effectiveness of a teaching module on participants. Now, research has found its way much broader than imagination. It involves narrative reviews, clinical trials, systematic and scoping reviews and even metaanalyses. The results are evidence-based and implemented immediately in the patient care modules. ${ }^{16,17}$

At the same time, nurses have barriers from organization, learning and participating in research works, knowing about the scope of databases and differentiating between predator and genuine journals. This needs extreme effort and adequate resources and sessions to enable future nurses to conduct research studies and disseminate the results with scientific rationales. Konwar et al. found in their study that nurses had reluctant behaviour to search and find new literature of review. Also, they highlighted barriers from organization, either lack of resources or less authority and lack of cooperation by nurses were experienced. ${ }^{18}$

\subsection{Enhancement of faculty development}

Well qualified and competent teaching faculty only can prepare the best nurses for tomorrow. Faculty development word acts like an umbrella term under which many programs can be held, such as research activities, teaching, proctoring and administrative activities. ${ }^{19}$ Increasing demands from institutes, universities and UGC requirements have created tougher competition among faculty to reach higher levels. ${ }^{20}$ Nursing faculty now require more updated skills and active performance in various activities added to their credentials than just the eligibility criteria mentioned in the advertisements.

This challenge is highly demanding new learning on daily basis, and only those can acquire the place that keeps an eye on such requirements and works to meet the criteria need. Also, institutes should continuously incorporate structured programs for the regular enhancement of faculty development. ${ }^{21}$ Faculty vitality thus plays a great role here. D. Ignatavicius and C.E. Chung conducted a survey of faculty showing keen interest and desire to integrate new learning practices and strategies. They noted the autonomy 
of faculty as a barrier to curricular changes. ${ }^{22}$

\subsection{Standards and protocols by regulatory bodies}

Indian nursing council has considerable contribution but still has a long way to go in upgrading the profession. Regulatory bodies and associations must prioritise their work to reach goals related to the service of patients and better outcomes in society. Weak regulatory bodies in India, indeterminate regulatory functions, lack of amendment of acts, lack of representation of nurses and midwives in governing positions, poor quality of conferences conducted and approved, undermined leadership positions have impaired the growth of the profession. ${ }^{23}$

The Healthcare system should be capable of expanding well beyond even feasible capacity. Stronger the protocols developed by regulatory bodies for approval and accreditation of programs and mushrooming nursing colleges, effective will be the healthcare services provided to populations as per demands. Otherwise, meeting all needs of society would be a burden with greater shortages as expected.

\subsection{Cultural diversity}

Nursing care around the world to various groups of patients needs a good sense of understanding about their cultural practices and perceptions. Hence, there is a need of understanding this cultural diversity due to increased immigration and globalization to make sure evolving society will be satisfied with care. ${ }^{24}$

Diversity can also act as an opportunity for nurses, where working with different cultural backgrounds people and looking for their perspectives can be an educational venture for nursing students and nurses. This saves time and energy and helps to learn the needs of a patient in a clearer way by assessing and interpreting conveyed regards from patients. As student needs are evaluated and addressed more effectively, less time will be needed to clear up confusion and anger, less time will be spent in remediation, and less energy will be spent on frustration. ${ }^{25}$

\subsection{Economic concerns}

Nurses with fewer pay scales, even at the state and central level, promised fake incentives on work extracted from them, and extremely slow growth in funds they receive for official works are the biggest challenges now. This is the reason that is encouraging nurses to immigrate to foreign countries for better economic survival. India still has discrepancies in paying nurses compared to other employees of different disciplines with equal qualifications. ${ }^{26}$

A transparent system of accounts and facilities available for nurses should exist, and it should be made known to the workforce to avail the benefits properly. This motivates nurses for better outcomes of institutes and provides scholarships, sponsorship for intellectuals for higher studies, allowing opportunities to take up fellowships and post doctoral programs would help to grow the profession to a better extent and create strong leaders in the profession.

\section{Conclusion}

The historical viewpoint of nursing education has advanced over the past 100 years. During the $19^{t h}$ and $20^{t h}$ centuries, the main goal of nursing education was to enable the nurses to assist the physicians. Now, the nurses are shouldering up a new sense of responsibility in multiple areas. Nursing education is seeing multiple facets of reformation around the world, expecting nurses with expanded roles to be performed. This needs adequate preparation of faculty and students by proper development opportunities. And nurse educators should learn to take advantage of the latest technology and provide more effective and efficient teaching and skills to students. Assessment of periodical anticipated obstacles and recognizing the ways to approach them safeguards patient care and brings satisfaction in student nurses.

To tackle these demands, a tie-up between educational institutes and tertiary care hospitals would be a better idea. Identifying the nurses in service with extraordinary interest and efficient skills and providing them with a platform to mentor and supervise educational activities in clinical settings can contribute a remarkable path ahead. Strengthening regulatory bodies for better implementation of policies and evaluation system needs an arduous effort by nurse executives and leaders.

\section{Conflict of Interest}

None.

\section{References}

1. Nursing Practice and Service and the Social and Economic Welfare of Nurses. Am J Nurs. 1969;69(10):2177-9.

2. Habibi S, Hachesoo PR, Tabaghi R. Enhancing information literacy as a base of developing evidence-based nursing. Heal Inf Manag. 2010;7(3):371-8.

3. Hung HY, Wang YW, Feng JY, Wang CJ, Lin ECL, Chang YJ, et al. Evidence-based practice curriculum development for undergraduate nursing students: The preliminary results of an action research study in Taiwan. J Nurs Res. 2019;27(4):e30.

4. Ilic D. Teaching evidence-based practice: perspectives from the undergraduate and post-graduate viewpoint. Ann Acad Med Singap. 2009;38(6):559-65.

5. Fitzpatrick JJ. The International Council of Nurses, the NLN, and you and I. Nurs Educ Perspect. 2009;30(3):145-6.

6. Borger AL. The institute of medicine and the future of nursing. $J$ Dermatol Nurses Assoc. 2012;4(2):87-8.

7. Gale J, Ooms A, Grant R, Paget K, Marks-Maran D. Student nurse selection and predictability of academic success: The Multiple Mini Interview project. Nurse Educ Today. 2016;40:123-30.

8. Jamshidi N, Molazem Z, Sharif F, Torabizadeh C, Kalyani MN. The Challenges of Nursing Students in the Clinical Learning Environment: 
A Qualitative Study. Sci World J. 2016;2016:1-7.

9. Miguel PJ, Puga MP, Ana R, Jose R, and CP. Clinical Virtual Simulation in Nursing Education: Randomized Controlled Trial. $J$ Med Internet Res. 2019;21(3): 11529.

10. Grading System | GPA | CGPA | Advantages | Disadvantages [Internet]. [cited 2021 Oct 11]. Available from: https://targetstudy. com/articles/grading-system.html.

11. Jacobs GM, Farrell TSC. Paradigm shift: Understanding and implementing change in second language education; 2001.

12. Eyikara $\mathrm{E}$, and ZGB. The importance of simulation in nursing education. World J Educ Technol Curr Issues. 2017;9(1):2-7.

13. Prasuna J, Sharma R, Osce. TEM: Different approaches to assess clinical skills of nursing students. Iran J Nurs Midwifery Res. 2017;22(1):78-80.

14. Goswami G, Sharma SK, Sharma R, R R. Simulation and skill training facilities in nursing institutes at Uttarakhand: A cross-sectional study. Iran J Nurs Midwifery Res. 2021;26(5):454-454.

15. Sharma R, Mohanty A, Singh V, Va S, Gupta PK, Jelly P. Effectiveness of Video-Based Online Training for Health Care Workers to Prevent COVID-19 Infection: An Experience at a Tertiary Care Level Institute. vol. 13. Uttarakhand, India. Cureus; 2021. p. 14785-14785.

16. Burns PB, Rohrich RJ, Chung KC. The Levels of Evidence and their role in Evidence-Based Medicine. Plast Reconstr Surg. 2011;128(1):310.

17. Glasofer A, Townsend AB. Determining the level of evidence: Experimental research appraisal. Nurs Crit Care. 2019;14(6):22-5.

18. Konwar G, Kalita J. The Barriers and Challenges of Conducting Nursing Research and Communicating Research Findings into Practice. Int J Heal Sci Res. 2018;8(6):222.

19. Institute of Medicine (US) Committee on the Robert Wood Johnson Foundation Initiative on the Future of Nursing at the Institute of Medicine. Washington (DC; US): National Academies Press; 2011. Available from: https://www.ncbi.nlm.nih.gov/books/NBK209885/.

20. University Grants Commission, editor. Quality Mandate for Higher Education Institutions in India [Internet]. New Delhi: Secretary, University Grants Commission; 2021 [cited 2021 Oct 11]. Available from: https://www.ugc.ac.in/e-book/QualityMandateE-BOOK.pdf.
21. Bilal SG, Chen S. The impact and effectiveness of faculty development program in fostering the faculty's knowledge, skills, and professional competence: A systematic review and meta-analysis. Saudi J Biol Sci. 2019;26(4):688-97.

22. Ignatavicius D, Chung CE. Professional Development for Nursing Faculty: Assessing Transfer of Learning into Practice. Teach Learn Nurs. 2016;11(4):138-42.

23. Mayra K, Padmadas SS, Matthews Z. Challenges and needed reforms in midwifery and nursing regulatory systems in India: Implications for education and practice. PLoS One. 2021;16(5):e0251331.

24. Murcia SEA, Lopez L. The experience of nurses in care for culturally diverse families: A qualitative meta-synthesis. Rev Lat Am Enfermagem. 2016;24:e2718.

25. Fawaz MA, Hamdan-Mansour AM, Tassi A. Challenges facing nursing education in the advanced healthcare environment. Int $J$ Africa Nurs Sci. 2018;9:105-10.

26. Palmer SP. Nurse retention and satisfaction in Ecuador: implications for nursing administration. J Nurs Manag. 2014;22(1):89-96.

\section{Author biography}

Rakesh Sharma, Assistant Professor

Shweta Garadi, Nursing Tutor

Prasuna Jelly, Assistant Professor

Ruchika Rani, Assistant Professor

Cite this article: Sharma R, Garadi S, Jelly P, Rani R. Transformation in nursing education in India: Current issues. IP Int J Med Paediatr Oncol 2021;7(3):115-118. 